\title{
4-Step Total Synthesis of (-)-Pavidolide B
}

\author{
Yaoqiu Zhu, ${ }^{*}$ Elkin L. Romero, ${ }^{\dagger}$ Kolluru Srinivas,${ }^{\dagger}$ Elizabeth Noriega
}

\begin{abstract}
Department of Chemistry and Biochemistry, Border Biomedical Research Center, The University of Texas at El Paso, TX 79968, United States
\end{abstract}

†These authors contributed equally.

\author{
Supporting Information Placeholder
}

\begin{abstract}
Pavidolide B is a complex tetracyclic diterpenoid with seven contiguous stereocenters, and classical total syntheses have recently been reported. In this work, an annulation strategy of carbanion cascade reaction is formulated for accomplishing an asymmetric total synthesis of (-)-pavidolide B in just 4 linear steps. The key reaction is a tandem intramolecular Michael addition, which constructs two 5-membered rings and five stereocenters in a highly selective fashion in one step. This work represents one of the most efficient cases of total synthesis and demonstrates that synthetic access to complex therapeutic natural product can be greatly simplified.
\end{abstract}

Diterpenoid (-)-pavidolide B (Figure 1) was isolated from the marine soft coral Sinularia pavida by Lin and co-workers in 2012. ${ }^{1}$ In initial biological studies, the natural product has demonstrated selective inhibitory activity against human promyelocytic leukemia cell line HL-60. The complex structure of (-)-pavidolide B is characterized by a $6 / 5 / 5 / 7$ fusedring system containing seven contiguous stereocenters, which poses significant challenges to organic synthesis. In 2017, Yang and co-workers reported the first total synthesis that features some stunning and non-intuitive approaches. ${ }^{2}$ Starting from $(S)$-carvone, the other three rings of the tetracyclic structure were constructed through a series of sophisticated reactions, which include an enantioselective organocatalytic cyclopropanation, a radical-based regio- and diastereo-selective cascade annulation, a sequential ring-closing metathesis and a final $\mathrm{RhCl}_{3}$-catalyzed double bond migration and inversion of the C11 stereocenter. Subsequently, Ding and co-workers reported their total synthesis work based on classical ring constructions and elegant ring rearrangement. 3,4

With an interest in bioinspired total synthesis, we examined the intriguing structure of (-)-pavidolide B to seek alternative synthetic routes (Figure 1). Among the tetracyclic system, both the 6-membered A-ring and the 7-membered Dring contain ketone functionalities, and these two rings are fused to form the 5 -membered $\mathrm{C}$-ring with their $\alpha, \beta$-carbon bonds of the ketone functionality (i.e., $\mathrm{C}_{4}-\mathrm{C}_{13}$ and $\mathrm{C}_{11}-\mathrm{C}_{5}$, respectively) together with the ester $\alpha$-carbon (C12) in the Bring. This suggests that the B-ring and C-ring can be formed through a tandem sequence of two Michael additions. $5^{-8}$ As illustrated in Figure 1, in the structure of the 'A-D ester' intermediate, the deprotonated $\alpha$-carbon (C12) of the ester group can attack the $\alpha, \beta$-unsaturated ketone in the A-ring; after the first addition, the resulting carbanion on the A-ring can attack the $\alpha, \beta$-unsaturated ketone in the D-ring for the second addition. The tandem intramolecular Michael reaction can not only construct the B-ring and C-ring in just one step, but might also extend the chirality of the $(S)$-carvonederived A-ring to achieve the desired configurations of the other five stereocenters.

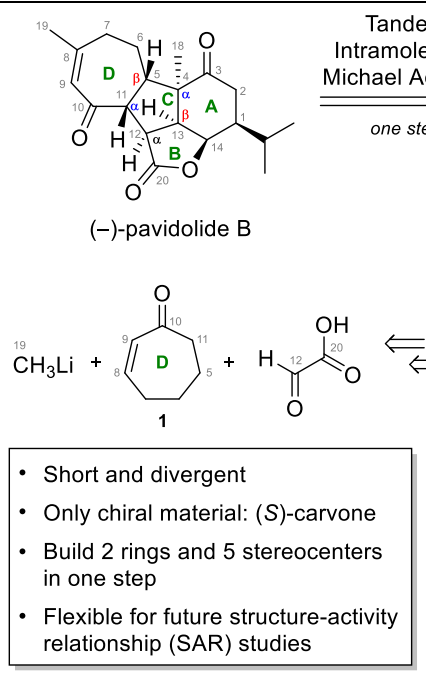

Figure 1. Retrosynthetic analysis of (-)-pavidolide B.

The desired A-D ester can be obtained from a Mitsunobu reaction between an A-ring alcohol fragment and a D-ring carboxylic acid fragment (Figure 1). The preparation of the Aring alcohol fragment from $(S)$-carvone has been well documented. ${ }^{2,9}$ The D-ring fragment is another ten-carbon moiety with a carboxylic acid functionality. Although this structure can be biosynthetically traced to two molecules of levulinic acid, it will be challenging to prepare it through condensation reaction of levulinates due to tedious stereochemical control. With the consideration of synthetic flexibility for future structure-activity relationship (SAR) studies, we decided to build the D-ring fragment from conjugated cycloheptenone (1).

The synthesis commenced with the preparation of the Dring carboxylic acid intermediate. As shown in Scheme 1, the first step of $\mathbf{1}$ to $\mathbf{3}$ conversion is accomplished by consolidating the reported two-step procedures, ${ }^{10,11}$ and the preparation can be conveniently performed in gram-scale in $71 \%$ yield, which is higher than the combined yields of the 2-step methods (30-50\%). In the second step, an acid-catalyzed direct condensation reaction between $\mathbf{3}$ and glyoxylic acid was conducted to afford the carboxylic acid intermediate $\mathbf{4 - E}$ in $30 \%$ yield. The low yield of this step is likely due to the de- 
composition of the conjugated ketone $\mathbf{3}$ under heating condition. Although multistep procedures, e.g. LDA-facilitated 3 condensation with ethyl glyoxylate followed by dehydration and ester hydrolysis, were adopted for yield improvement, this one-step direct condensation can be easily carried out in gram-scale and proves to be robust and efficient for achieving the goal of facile total synthesis. We have attempted to use bases such as DBU to isomerize the exocyclic double bond in 4-E to the desired endocyclic position but did not succeed, which might be due to the interference from the carboxylic acid group. Subsequently, we decided to use $4-\boldsymbol{E}$ to prepare the A-D ester intermediate and isomerize the double bond in the ester form. Following well-documented procedures, ${ }^{2,9}$ the A-ring alcohol intermediate 6 was prepared from $(S)$-carvone in two steps.

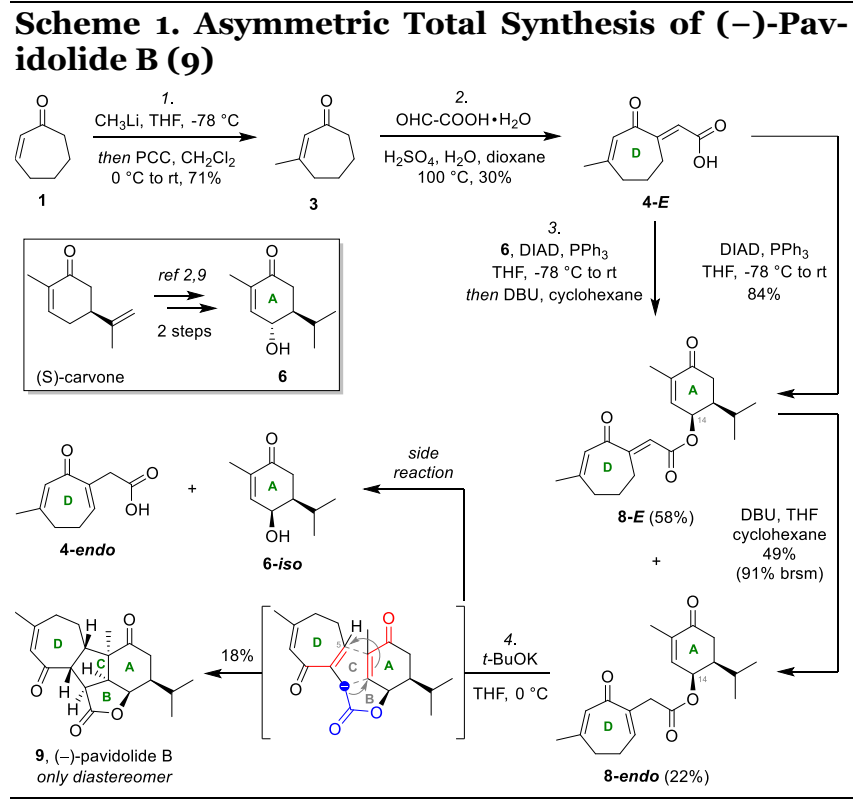

As shown in Scheme 1, the carboxylic acid fragment $4-E$ and the alcohol fragment $\mathbf{6}$ were initially condensed via a Mitsunobu reaction to form ester $\mathbf{8 - E}$ in $84 \%$ yield, and $\mathbf{8 - E}$ can be isomerized at room temperature by DBU in cyclohexane to afford the desired 8-endo in $49 \%$ yield ( $91 \% \mathrm{brsm}$ ). HPLC studies show that this mild condition does not epimerize the $\gamma$-position of the carvone moiety (C14). We then consolidated these two reactions into the 'one-pot' third step, which readily affords the two isomers, 8-endo and 8-E, in $22 \%$ and $58 \%$ yield, respectively. Higher temperatures were tested for the DBU-promoted isomerization but the intermediates were found to be unstable over $50{ }^{\circ} \mathrm{C}$. Although this double bond migration is not highly efficient due to the nature of the equilibrium, the two isomers can be conveniently separated, and the recovered 8-E can be converted to 8endo again through the facile protocol. To channel the final step of the intramolecular Michael reaction, we first tried Na$\mathrm{OMe}$ in anhydrous THF at $\mathrm{O}^{\circ} \mathrm{C}$, and only ester cleavage was observed. We then replaced $\mathrm{NaOMe}$ with $t$-BuOK. After two hours at $0{ }^{\circ} \mathrm{C}$ and a quick workup, ${ }^{1} \mathrm{H}$ NMR of the crude product shows the presence of $\mathbf{9}$ together with the ester cleavage product 6-iso (Scheme 1) and some other inconclusive side products. The ratio of $\mathbf{9}$ to $\mathbf{6}$-iso was initially found to be 1 to 2.6 and was subsequently improved to 1 to 2.0 when the reaction was conducted in glovebox. In the attempts to purify the crude mixture on silica gel, $\mathbf{9}$ was found to closely elute with 6-iso. We then used HPLC for further separation. Upon final purification, the product shows completely matching ${ }^{1} \mathrm{H}$ and ${ }^{13} \mathrm{C}$ NMR spectra to those reported for (-)-pavidolide
$\mathrm{B},,^{1,2}$ which verifies that $t$-BuOK can promote the carbanion cascade annulation of $\mathbf{8 - e n d o}$ to yield the complex natural product 9, albeit at a relatively low purification yield of $18 \%$.

For the optimization of the last step, we conducted condition screenings and used ${ }^{1} \mathrm{H}$ NMR of the crude reaction mixture for evaluation (Table 1). First we tested different bases and found only potassium bases, i.e., $t$-BuOK or KHMDS, can promote the desired reaction. When KHMDS was used, the reaction finished in one hour, and ${ }^{1} \mathrm{H}$ NMR of the crude product shows a less favorable ratio of 9 to 6-iso (1 to 3.1). We then tested different temperatures for the $t$-BuOK reaction and observed no turnover at low temperatures $\left(-78\right.$ or $\left.-20{ }^{\circ} \mathrm{C}\right)$ and quick decomposition at room temperature. Following these results, we tested substoichiometric amount of $t$-BuOK, a couple of Lewis acid additives and different solvents at $0^{\circ} \mathrm{C}$ but did not observe any improvement. For example, when 0.5 equivalents of $t$-BuOK were used, the reaction was found to be very slow. After six hours, only trace amount of 9 was observed while 8-endo remains majorly unchanged (no ester cleavage product was observed).

Table 1. Reaction condition studies of 8-endo annulation to (-)-Pavidolide B (9)

\begin{tabular}{|c|c|c|c|c|}
\hline entry $^{a}$ & base, additive & solvent & temp $\left({ }^{\circ} \mathrm{C}\right)$ & results \\
\hline 1 & $\mathrm{NaOMe}$ & THF & 0 & ester cleavage only \\
\hline 2 & $\mathrm{NaH}$ & THF & o & complex mixture \\
\hline $3^{b}$ & KHMDS & THF & o & $1: 3.1^{c}$ \\
\hline 4 & LDA & THF & -78 & no reaction \\
\hline $5^{d}$ & $t$-BuOK & THF & -78 & no reaction \\
\hline $6^{d}$ & $t$-BuOK & THF & -20 & no reaction \\
\hline $7^{e}$ & $t$-BuOK & THF & o & $1: 2.0^{c, f}$ \\
\hline $8^{b}$ & $t$-BuOK & THF & 23 & decomposed \\
\hline $9^{d}$ & $t-\mathrm{BuOK}^{g}$ & THF & o & slow reaction ${ }^{h}$ \\
\hline 10 & $t$-BuOK, $\mathrm{Cu}(\mathrm{OTf})_{2}{ }^{i}$ & THF & o & no reaction \\
\hline 11 & $t$-BuOK, $\mathrm{FeCl}_{3}{ }^{i}$ & THF & o & no reaction \\
\hline 12 & $t$-BuOK & 1,4-dioxane & o & no reaction \\
\hline 13 & $t$-BuOK & DCM & o & no reaction \\
\hline 14 & $t$-BuOK & diethyl ether & o & no reaction \\
\hline 15 & $t$-BuOK & toluene & o & no reaction \\
\hline
\end{tabular}

${ }^{a}$ Four hours and 1.2 equiv. of base were employed for all the reactions. ${ }^{b}$ Reaction time is 1 h. ${ }^{c}$ Ratio of 9 to 6-iso. ${ }^{d}$ Reaction time is 6 h. ${ }^{e}$ Reaction time is 2 h. ${ }^{f} \mathrm{HPLC}$ purification yield is $10 \% .{ }^{g} \mathrm{O} .5$ equiv. of $t$-BuOK were used. ${ }^{h 1} \mathrm{HNMR}$ shows 8-endo:9 of 15:1. ${ }^{i} 1$.o equiv. of Lewis acid was used.

It should be noted that we used freshly distilled solvents as well as anhydrous reagents and also performed the reactions in glovebox but could not diminish the side reactions that accompany the intramolecular annulation (Scheme 1). The ester cleavage side reaction yields 6-iso and 4-endo, and the latter was confirmed in the reaction mixture prior to workup by high resolution mass spectral (HRMS) results including fragmentation analysis. On the basis of these observations, we have proposed a mechanism for this side reaction. As shown in Scheme 2, the ester $\alpha$-carbon of 8-endo undergoes deprotonation, and the resulting enolate might proceed a 'ketene-elimination' pathway, leading to ester cleavage. The planar enolate substructure of the short ester link is conferred with conformational strain between the two rings and steric hindrance from the adjacent cis-isopropyl group on the Aring, and its nucleophilic attack to the $\alpha, \beta$-unsaturated ketone in the A-ring should require relatively high activation energy, which might also promote the elimination side reaction. To test this proposed ketene pathway, we quenched the anhydrous THF reaction at two hours upon the completion of the reaction with a mixture of $\mathrm{MeOH}$ and $\mathrm{H}_{2} \mathrm{O}(1 / 1, \mathrm{v} / \mathrm{v})$, and HRMS studies including fragmentation analysis de- 
tected the methyl ester form of 4-endo (Scheme 2). Although an ester-hydrolysis pathway cannot be fully ruled out, the $\mathrm{MeOH} / \mathrm{H}_{2} \mathrm{O}$-quench study results together with the observation that 8-endo is stable in THF in the presence of 0.5 equivalents of $t$-BuOK at $0{ }^{\circ} \mathrm{C}$ for six hours (Table 1) support the proposed 'ketene-elimination' pathway.

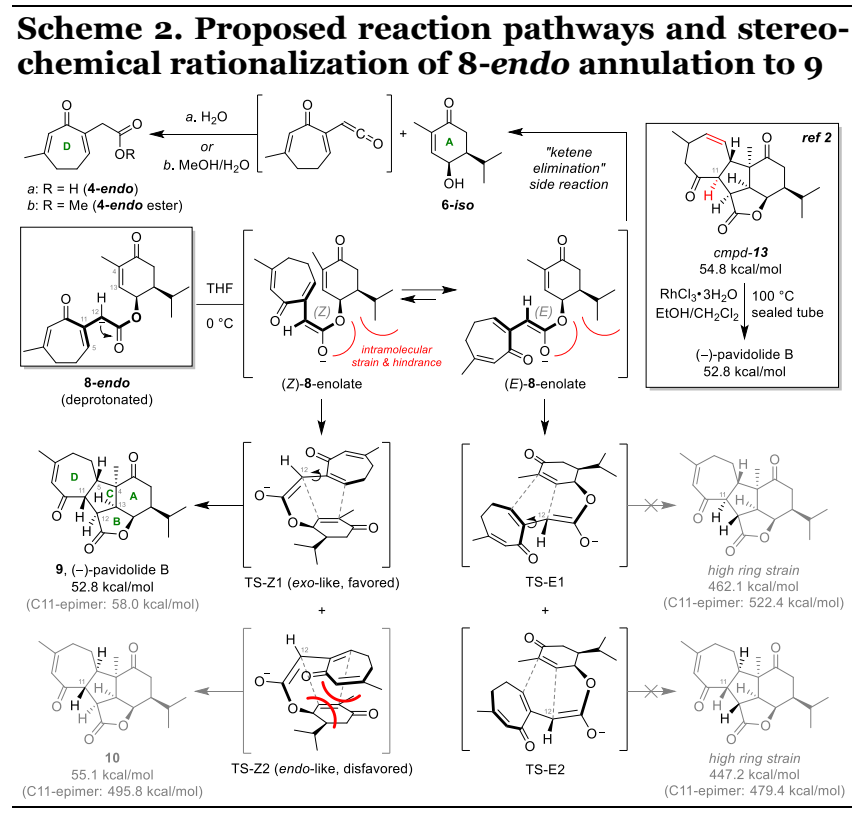

Despite of the ester cleavage side reaction, 9 was detected as the only diastereomeric product, which echoes the last step of Yang's pioneer synthesis, i.e., a $\mathrm{RhCl}_{3}$-promoted isomerization of $\mathrm{cmpd}-\mathbf{1 3}$ at $100{ }^{\circ} \mathrm{C}$ to yield (-)-pavidolide $\mathrm{B}$ (9) as the most stable diastereomer (Scheme 2, inserted). ${ }^{2}$ With the rigid tetracyclic structures, the relative stabilities of 9 and its diastereomers can be reliably assesses by total energy calculation. ${ }^{12}$ For example, the calculated total energy of $9(52.8 \mathrm{kcal} / \mathrm{mol})$ is lower than that of cmpd-13 $(54.8$ $\mathrm{kcal} / \mathrm{mol}$ ), which is consistent with the reported thermodynamic isomerization. ${ }^{2}$ The high stereochemical selectivity observed in the intramolecular annulation of 8-endo can be rationalized along the tandem formation sequence of the five stereocenters, i.e., C12-C13-C4-C5-C11 of the C-ring (Scheme 2). Upon deprotonation, the intermediate of $\mathbf{8}$-enolate can adapt either the $(Z)$ - or $(E)$-conformation. Upon nucleophilic attack from $\mathrm{C}_{12}$, the next three stereocenters ( $\left.\mathrm{C}_{13}-\mathrm{C}_{4}-\mathrm{C}_{5}\right)$ are naturally decided by the relative positions of the two fusing rings. When A-ring and D-ring fuse to the rigid structure, the last stereocenter $\mathrm{C} 11$ resorts to its more stable epimer configuration, from the enolate intermediate. As shown in Scheme 2, $(Z)$ - or $(E)$-8-enolate can each adopt two transitional states for annulation. Although $(E)$-8-enolate is of lower energy than (Z)-8-enolate, the high ring strains of the annulation products yielded from TS-E1 and TS-E2 (> $400 \mathrm{kcal} / \mathrm{mol})$ exclude these paths. $(Z)-8$-enolate can adopt an exo-like transition state (TS-Z1) to yield the stable product $\mathbf{9}$; it can also adopt an alternative endo-like transition state (TS-Z2) to yield diastereomer $10(55.1 \mathrm{kcal} / \mathrm{mol})$. However the steric clash of TS-Z2 precludes this path leading to the formation of 10. Despite of the fact that 9 is the only diastereomeric product, the desired intramolecular annulation needs to proceed with the less stable $(Z)-8$-enolate intermediate and requires energy to overcome the conformational obstacles and steric hindrances, which can also promote side reactions. Overall, the tandem intramolecular Michael addition of 8-endo is an intriguing transformation: on the one hand, it is enabled by the linear assembly of 'one ester between two $\alpha, \beta$-unsaturated ketones'; on the other hand, the tight chemical spaces pose significant constrains to the intramolecular annulation and confers major side reactions.

Natural product total synthesis is arguably the most challenging subject in chemistry. For example, the synthetic access to the terpenoid family that represents the epitome of molecular complexity often requires not only a daunting number of steps but also superb craftsmanship to perform these steps. In nature, tandem cascade of carbocation reaction of nonpolar $\mathrm{C}-\mathrm{C}$ double bonds are important biosynthetic pathways of cyclic terpenes, whose carbon skeletons undergo subsequent enzyme-mediated oxygenation to form diversified terpenoids. ${ }^{13,14}$ The strategy of tandem intramolecular Michael addition, termed as TIMA, blends the two biosynthetic processes, through cascade reactions of oxygenstabilized carbanions with oxygen-polarized $\mathrm{C}-\mathrm{C}$ double bonds. Through literature survey, we have identified precedent that has demonstrated the similar concept, 15,16 and our work reported here supports TIMA to be a highly efficient synthetic methodology for building complex ring systems in organic synthesis.

In conclusion, a facile and protecting-group-free asymmetric total synthesis of (-)-pavidolide $B$ is accomplished in a total of only 6 steps or 4 linear steps. The efficient synthetic access to this complex marine diterpenoid is enabled by a tandem intramolecular Michael addition, which constructs two fused 5-membered rings and five contiguous stereocenters in one step. This annulation strategy can be applied to guide the design and synthesis of other challenging organic structures. The divergent and flexible route can also aid the exploration of the anticancer mechanism of the natural product as well as channel future SAR studies for discovering potent and selective chemotherapeutics.

\section{ASSOCIATED CONTENT}

\section{Supporting Information}

The Supporting Information is available free of charge on the ACS Publications web site at.

Experimental procedures and spectroscopic data of compounds, NMR spectra of compounds, comparison of the spectra and data (PDF).

\section{AUTHOR INFORMATION}

\section{Corresponding Author}

*yzhu2@utep.edu

\section{Author Contribution}

${ }^{\dagger}$ E.L.R. and K.S. contributed equally.

\section{Notes}

The authors declare no competing financial interest.

\section{ACKNOWLEDGMENT}

We thank Dr. H. Wang (Sorrento Therapeutics), Dr. Q. Cai (Jinan University) and Dr. H. Zhao (AbbVie, Inc.) for helpful discussions. Financial support was provided by the Border Biomedical Research Center (National Institutes of Health, 5G12MDo07592) and the University of Texas at El Paso.

\section{REFERENCES}

(1) Shen, S.; Zhu, H.; Chen, D.; Liu, D.; van Ofwegen, L.; Proksch, P.; Lin, W. Tetrahedron Lett. 2012, 53, 5759-5762. 
(2) Zhang, P. P.; Yan, Z. M.; Li, Y. H.; Gong, J. X. Yang, Z. J. Am. Chem. Soc. 2017, 139, 13989-13992.

(3) He, C.; Xuan, J.; Rao, P.; Xie, P. P.; Hong, X.; Lin, X.; Ding, H. Angew. Chem. Int. Ed. 2019, 58, 5100-5104.

(4) Rao, P.; Hu, J.; Xuan, J.; Ding, H. J. Org. Chem. 2o19, 84, 9385-9392.

(5) Ihara, M.; Toyota, M.; Fukumoto, K.; Kametani, T. Tetrahedron Lett. 1984, 25, 3235-3238.

(6) Hagiwara, H.; Yamada, Y.; Sakai, H.; Suzuki, T.; Ando, M. Tetrahedron 1998, 54, 10999-11010.

(7) Amat, M.; Arioli, F.; Pérez, M.; Molins, E.; Bosch, J. Org. Lett. 2013, 15, 2470-2473.

(8) Lu, H.-H.; Martinez, M. D.; Shenvi, R. A. Nat. Chem. 2015, 7, 604-607.
(9) García-Cabeza, A. L.; Marín-Barrios, R.; Azarken, R.; MorenoDorado, F. J.; Ortega, M. J.; Vidal, H.; Gatica, J. M.; Massanet, G. M.; Guerra, F. M. Eur. J. Org. Chem. 2013, 36, 8307-8314.

(10) Kehrli, S.; Martin, D.; Rix, D.; Mauduit, M.; Alexakis, A. Chem. Eur. J. 2010, 16, 9890-9904.

(11) Mudryk, B.; Cohen, T. J. Am. Chem. Soc. 1993, 115, 3855-3865. (12) Energy calculation is performed using ChemBio3D Ultra using MM2 method.

(13) Tantillo, D. J. Nat. Prod. Rep. 2011, 28, 1035-1053.

(14) Koksal, M.; Jin, Y.; Coates, R. M.; Croteau, R.; Christianson, D. W. Nature 2011, 469, 116-120.

(15) Takasu, K.; Mizutani, S.; Noguchi, M.; Makita, K.; Ihara, M. J. Org. Chem. 2000, 65, 4112-4119.

(16) Xue, H.; Gopal, P.; Yang, J. J. Org. Chem. 2012, 77, 89338945 .
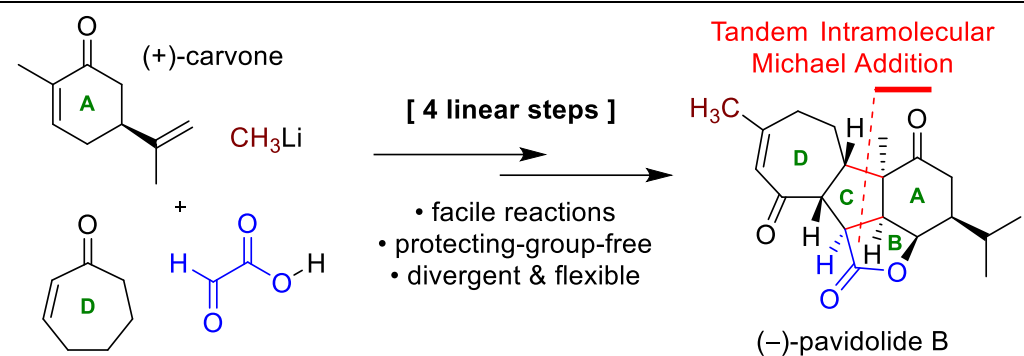

Graphical abstract 